\title{
Improving assessment of daily energy expenditure by identifying types of physical activity with a single accelerometer.
}

Citation for published version (APA):

Bonomi, A., Plasqui, G., Goris, A. H., \& Westerterp, K. R. (2009). Improving assessment of daily energy expenditure by identifying types of physical activity with a single accelerometer. Journal of Applied Physiology, 107(3), 655-661. https://doi.org/10.1152/japplphysiol.00150.2009

Document status and date:

Published: 01/01/2009

DOI:

10.1152/japplphysiol.00150.2009

Document Version:

Publisher's PDF, also known as Version of record

Document license:

Taverne

Please check the document version of this publication:

- A submitted manuscript is the version of the article upon submission and before peer-review. There can be important differences between the submitted version and the official published version of record.

People interested in the research are advised to contact the author for the final version of the publication, or visit the DOI to the publisher's website.

- The final author version and the galley proof are versions of the publication after peer review.

- The final published version features the final layout of the paper including the volume, issue and page numbers.

Link to publication

\footnotetext{
General rights rights.

- You may freely distribute the URL identifying the publication in the public portal. please follow below link for the End User Agreement:

www.umlib.nl/taverne-license

Take down policy

If you believe that this document breaches copyright please contact us at:

repository@maastrichtuniversity.nl

providing details and we will investigate your claim.
}

Copyright and moral rights for the publications made accessible in the public portal are retained by the authors and/or other copyright owners and it is a condition of accessing publications that users recognise and abide by the legal requirements associated with these

- Users may download and print one copy of any publication from the public portal for the purpose of private study or research.

- You may not further distribute the material or use it for any profit-making activity or commercial gain

If the publication is distributed under the terms of Article $25 \mathrm{fa}$ of the Dutch Copyright Act, indicated by the "Taverne" license above, 


\title{
Improving assessment of daily energy expenditure by identifying types of physical activity with a single accelerometer
}

\author{
A. G. Bonomi, ${ }^{1,2}$ G. Plasqui, ${ }^{1}$ A. H. C. Goris, ${ }^{3}$ and K. R. Westerterp ${ }^{1}$ \\ ${ }^{1}$ Department of Human Biology, Maastricht University, Maastricht; and ${ }^{2}$ Group Care and Health Applications \\ and ${ }^{3}$ DirectLife New Wellness Solutions, Philips Research Laboratories, Eindhoven, The Netherlands
}

Submitted 11 February 2009; accepted in final form 23 June 2009

\begin{abstract}
Bonomi AG, Plasqui G, Goris AH, Westerterp KR. Improving assessment of daily energy expenditure by identifying types of physical activity with a single accelerometer. J Appl Physiol 107: 655-661, 2009. First published June 25, 2009; doi:10.1152/japplphysiol.00150.2009.Accelerometers are often used to quantify the acceleration of the body in arbitrary units (counts) to measure physical activity (PA) and to estimate energy expenditure. The present study investigated whether the identification of types of PA with one accelerometer could improve the estimation of energy expenditure compared with activity counts. Total energy expenditure (TEE) of 15 subjects was measured with the use of doublelabeled water. The physical activity level (PAL) was derived by dividing TEE by sleeping metabolic rate. Simultaneously, PA was measured with one accelerometer. Accelerometer output was processed to calculate activity counts per day $\left(\mathrm{AC}_{\mathrm{D}}\right)$ and to determine the daily duration of six types of common activities identified with a classification tree model. A daily metabolic value $\left(\mathrm{MET}_{\mathrm{D}}\right)$ was calculated as mean of the MET compendium value of each activity type weighed by the daily duration. TEE was predicted by $\mathrm{AC}_{\mathrm{D}}$ and body weight and by $\mathrm{AC}_{\mathrm{D}}$ and fat-free mass, with a standard error of estimate (SEE) of $1.47 \mathrm{MJ} /$ day, and 1.2 $\mathrm{MJ} /$ day, respectively. The replacement in these models of $\mathrm{AC}_{\mathrm{D}}$ with $\mathrm{MET}_{\mathrm{D}}$ increased the explained variation in TEE by $9 \%$, decreasing SEE by $0.14 \mathrm{MJ} /$ day and $0.18 \mathrm{MJ} /$ day, respectively. The correlation between $\mathrm{PAL}_{\text {and }} \operatorname{MET}_{\mathrm{D}}\left(R^{2}=51 \%\right)$ was higher than that between PAL and $\mathrm{AC}_{\mathrm{D}}$ $\left(R^{2}=46 \%\right)$. We conclude that identification of activity types combined with MET intensity values improves the assessment of energy expenditure compared with activity counts. Future studies could develop models to objectively assess activity type and intensity to further increase accuracy of the energy expenditure estimation.
\end{abstract}

double-labeled water; motion sensor; classification tree; activity recognition

IN MANY METABOLIC DISORDERS there is a need to measure daily energy expenditure. The main determinants of energy expenditure are body size and physical activity (PA) (30). Although body size can be easily determined, the assessment of PA represents a challenge because of diversified individual behaviors and because of the complex nature of human activities. Several methods have been proposed to objectively measure PA (18). Ideally, PA should be measured in free-living conditions, over a period of time representative for the habitual activity level, and with minimal discomfort to the subject. Accelerometers reasonably satisfy these requirements and, therefore, have been used widely for the assessment of PA (16, 18). Traditionally, accelerometer output has been expressed as activity counts to quantify PA. This measure of the acceleration of the body is commonly defined as the area under the rectified acceleration signal measured over a fixed time interval

Address for reprint requests and other correspondence: A. G. Bonomi, Human Biology, Maastricht Univ., PO Box 616, 6200 MD Maastricht, The Netherlands (e-mail: a.bonomi@HB.unimaas.nl). like 1 min (4). Activity counts have been used to describe the pattern of PA, i.e., the frequency, the duration, and the intensity of PA. Furthermore, activity counts proved to be linearly related to the total energy expenditure (TEE), to the activityrelated energy expenditure (AEE), and to the physical activity level (PAL) as measured with the use of double-labeled water $(8,14,21)$. TEE is defined as the daily metabolic rate, while AEE corresponds to the portion of TEE consumed for PA. PAL is also commonly used to describe the amount of energy consumed for PA as a fraction of the energy required to maintain basal metabolic functions. Linear models have been developed to predict TEE and AEE, using activity counts and subject characteristics such as body weight as independent variables $(8,20)$. In contrast, when indirect calorimetry was used to assess the metabolic rate during specific activities, the relationship between the intraindividual variability in AEE and activity counts varied according to the type of activity (19). Similar to TEE and AEE, PAL has been repeatedly predicted by linear models based on activity counts. However, as shown for AEE, the relationship between PAL and activity counts depends on the type of activity (19). Thus prediction models that account for the type of activity performed could result in more accurate estimates of TEE, AEE, and PAL.

In recent years, accelerometers have been used in combination with classification models to identify types of PA by evaluating information (features) derived from the acceleration of the body $(3,9,22,28,32)$. Classification trees (9), neural networks (32), and hidden Markov models (22) are some of the classification models used to identify activity type. Zhang et al. (32) developed a neural network to identify up to 32 human movements, recording the acceleration of the body with five accelerometers. In more recent studies, the identification of activity types was based on the acceleration features measured with a single accelerometer $(9,12)$. However, the simplification of the measurement system, using one accelerometer, implied a decrease in the number of activities that could be accurately identified by the classification model.

In this study PA was measured with a single accelerometer during daily life in a population of healthy adults. Simultaneously, TEE was assessed with the gold standard technique of double-labeled water. The aim was to investigate whether the identification of activity type combined with a simple methodology to define activity type intensity could improve the estimation of TEE, AEE, and PAL compared with daily activity counts.

\section{METHODS}

Subjects. Fifteen healthy, nonsmoking adults (9 men and 6 women) were recruited by advertisement in local newspapers to participate in the study. The study was approved by the Ethics Committee of the 
Maastricht University Medical Center, and written informed consent was obtained from the participants.

Study design. Subjects reported to the laboratory on day 0 at 9:00 PM for an overnight stay in a respiration chamber. The study included a 2-wk observation period for the measurements of energy expenditure, from the morning of day 1 until the morning of day 15. PA was monitored from the morning of day 1 until the morning of day 6 .

Anthropometrics. Anthropometric measurements were taken in the morning after an overnight fast. Body mass (BM) was measured on an electronic scale (Mettler Toledo ID1 Plus, Giessen, Germany) to the nearest $0.01 \mathrm{~kg}$. Height was measured to the nearest $0.1 \mathrm{~cm}$ (SECA Mod.220, Hamburg, Germany). Body volume was determined by underwater weighing. During the underwater weighing, residual lung volume was measured with the helium dilution technique (Volugraph 2000, Mijnhardt, Bunnik, The Netherlands). Total body water (TBW) was determined with deuterium dilution, according to the Maastricht protocol (31). Body composition was calculated from body mass, body volume, and TBW with Siri's three-compartment model (25).

Sleeping metabolic rate. Sleeping metabolic rate (SMR) was measured during an overnight stay in the respiration chamber. The room measured $14 \mathrm{~m}^{3}$ and was equipped with bed, table, chair, freeze toilet, washing bowl, radio, television, and computer (24). Energy expenditure was calculated from $\mathrm{O}_{2}$ consumption and $\mathrm{CO}_{2}$ production according to Weir's formula (29). SMR was defined as the lowest observed energy expenditure for three consecutive hours during the night. Room temperature was held constant at $20 \pm 1{ }^{\circ} \mathrm{C}$.

Energy expenditure. TEE was measured with the use of doublelabeled water according to the Maastricht protocol (31). On the evening of day 0 , after the collection of a background urine sample, subjects drank a weighed amount of ${ }^{2} \mathrm{H}_{2}{ }^{18} \mathrm{O}$ such that baseline levels were increased by $100 \mathrm{ppm}$ for ${ }^{2} \mathrm{H}$ and $200 \mathrm{ppm}$ for ${ }^{18} \mathrm{O}$. Additionally, urine samples were collected in the morning (from second voiding) of day 1, day 8, and day 15 and in the evening of day 1, day 7, and day 14. AEE was measured as $(0.9 \times \mathrm{TEE})-\mathrm{SMR}$, assuming the diet-induced thermogenesis to be $10 \%$ of TEE. The mean PAL was calculated as TEE/SMR (20).

Physical activity monitoring. The motion sensor used was a modified version of the previously validated Tracmor (Philips Research, Eindhoven, The Netherlands) $(4,20)$. The device was equipped with a triaxial piezo-capacitive [micro-electro-mechanical system (MEMS)] acceleration sensor and recorded acceleration samples 20 times per second. The accelerometer measured $8 \times 3.5 \times 1 \mathrm{~cm}$ and weighed $34.8 \mathrm{~g}$, including the battery, and was placed at the lower back with an elastic belt. The $x$-, $y$-, and $z$-axes of the accelerometer were oriented along the vertical, medio-lateral, and antero-posterior directions of the body, respectively. PA was monitored for 5 consecutive days ( 2 weekend days and 3 weekdays). Subjects were instructed to wear the accelerometer during waking hours, except during showering and water activities. A diary was used to report periods in which the subject was not wearing the accelerometer during the day.

The raw acceleration signal was downloaded to a personal computer and processed for two purposes. The first purpose was to determine the number of activity counts scored daily. The total activity counts accumulated during the monitoring period was divided by the number of days to determine the average activity counts per day $\left(A C_{D}\right)$. Second, the raw acceleration signal was processed to identify types of PA performed during the day. The acceleration signal was segmented in nonoverlapping intervals of $6.4 \mathrm{~s}$. This segment length was selected because the accuracy of classification models used to identify activity types could decrease when the acceleration signal is analyzed in portions of shorter time length (3). In each segment of the acceleration and for each sensing axis, the following acceleration features were determined: average, standard deviation, peak-to-peak distance, and dominant frequency in the power spectral density. Because of the high accuracy in identifying activity types $(3,9)$, a classification tree algorithm was employed to evaluate the features and to classify the acceleration in one of six activity classes: "lie," sitting or standing (Sit-Stand), active standing (AS), "walk," "run," and "cycle." The AS class was defined to represent dynamic activities not related to ambulation performed in the standing position. The outcome of the classification tree allowed the definition of the duration of the six activity types during the monitoring period. The average daily duration $\left(\mathrm{AD}_{\mathrm{D}}\right)$ of each activity type was calculated as the total duration of each activity divided by the number of monitoring days. The $\mathrm{AD}_{\mathrm{D}}$ of lying was determined by integrating the sleeping time, as reported with the diary, to the time spent lying during waking hours.

The $\mathrm{AD}_{\mathrm{D}}$ of the identified activity types was used for the assessment of PA by defining a daily metabolic equivalent value $\left(\mathrm{MET}_{\mathrm{D}}\right)$. The $\mathrm{MET}_{\mathrm{D}}$ was calculated as the mean of the standard metabolic equivalent value (MET) of each activity type weighed by the $\mathrm{AD}_{\mathrm{D}}$, as shown in the equation below:

$$
\operatorname{MET}_{\mathrm{D}}=\frac{1}{k} \sum_{i=1}^{6} \operatorname{MET}^{i} \times \mathrm{AD}_{\mathrm{D}}^{i}
$$

where $i$ is an index that corresponds to each of the six activity types considered, $\mathrm{MET}^{i}$ is the standard MET value for activity $i, \mathrm{AD}_{D}^{l}$ is the average daily duration for activity $i$ (min/day), and $k$ represents the number of monitoring minutes during the day. According to the diaries, the nonwearing time during waking hours was removed from the data set. This operation was analogous to considering the $\mathrm{MET}_{\mathrm{D}}$ of the nonwearing time equal to the average $\mathrm{MET}_{\mathrm{D}}$ of the wearing time. The standard MET for each activity type was obtained from a published compendium of PA (1). Since the MET of walking, running, and cycling depends on movement speed, the speed of these activities was estimated by employing recently developed prediction models based on acceleration features (3). The speed of each walking, running, and cycling bout was measured and averaged over the monitoring period and over each subject to have an indication of which MET value would be more suitable to describe the average intensity of the walking, running, and cycling activities.

Classification tree. A classification tree is a model in which the classification process is defined by a sequence of logical conditions based on the features of the object being classified. The development of a classification tree comprises the selection of the features that are most useful for the classification and the definition of logical conditions to steer the classification. The classification tree used in the present investigation was developed with data collected during a supervised test conducted in a separate study with a population characterized by a broad range of weight, height, and age: 20 men and 20 women, weight $82 \pm 23(48-182) \mathrm{kg}$ [mean $\pm \mathrm{SD}$ (min-max)], height $1.71 \pm 0.09(1.49-1.97) \mathrm{m}$, age $41 \pm 16(23-70) \mathrm{yr}$, and body mass index (BMI) $28.1 \pm 7.1(18.6-53.9) \mathrm{kg} / \mathrm{m}^{2}$. The supervised test included activities such as lying, sitting, standing still, walking, running, cycling, washing dishes, and sweeping the floor. The acceleration collected during the dishwashing and floor sweeping activities was used to define the AS category. The acceleration collected during sitting and standing still activities was used to define the Sit-Stand category. These two activities have been grouped together to form a single category because the use of one accelerometer to measure PA did not allow accurate distinction of the sitting and standing still postures (3). Figure 1 shows the structure of the developed classification tree and the features selected for the identification of activity type. Table 1 shows the performance of the classification tree as tested on five subjects not included in the population used to develop the model (26). The development of the classification tree was conducted with the Weka machine learning toolkit (University of Waikato, Hamilton, New Zealand) (10). The processing scripts used for the feature calculations and for the validation of the decision tree were developed with Matlab (MathWorks, Natick, MA).

Statistical analysis. Simple linear regression was used to develop prediction models for PAL using $\mathrm{AC}_{\mathrm{D}}$ or $\mathrm{MET}_{\mathrm{D}}$ as independent variable. The Bland-Altman plot was used to determine the agreement 


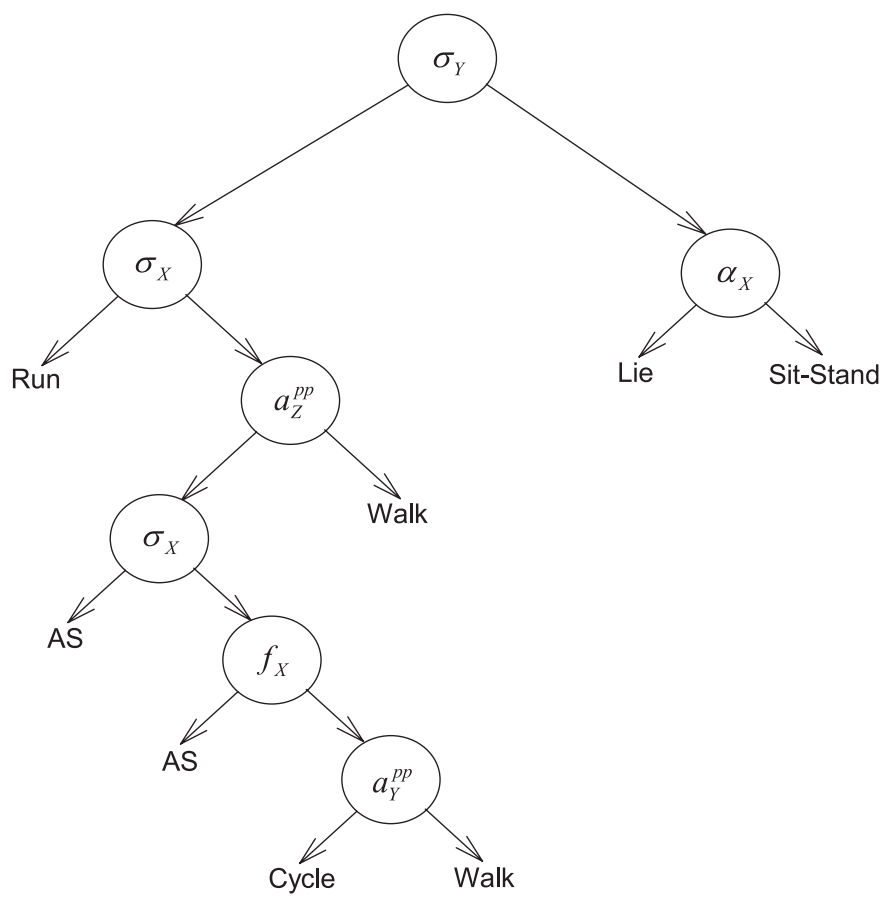

Fig. 1. Classification tree developed to identify types of physical activity. In the circles are noted the features used to identify activity types [lie, sit or stand (Sit-Stand), active standing (AS), walk, run, cycle]. The features selected for the classification were the standard deviation of the acceleration in the vertical and medio-lateral directions of the body $\left(\sigma_{X}, \sigma_{Y}\right)$, the average acceleration in the vertical direction of the body $\left(\alpha_{X}\right)$; the peak-to-peak distance of the acceleration measured in the medio-lateral and antero-posterior directions of the body $\left(a_{Y}^{\mathrm{pp}}, a_{Z}^{\mathrm{pp}}\right)$, and the frequency peak of the power spectral density of the acceleration measured in the vertical direction of the body $\left(f_{X}\right)$.

between measured and predicted PAL (2). Stepwise multiple linear regression analysis was used to select the best independent variables to predict TEE and AEE. Three different sets of independent variables were considered to account for the differences in body size: SMR, basic body characteristics (BM, height, age, and sex), and advanced body characteristics [fat mass, fat-free mass (FFM), age, sex]. The independent variable used to describe differences in PA was $\mathrm{AC}_{\mathrm{D}}$ or

Table 1. Performance of the model used to identify types of physical activity

\begin{tabular}{lrrrrrr}
\hline \hline & \multicolumn{5}{c}{ Classification Categories } \\
\cline { 2 - 7 } & Lie & Sit-Stand & AS & Walk & Run & Cycle \\
\hline True categories & & & & & & \\
$\quad$ Lie & 100 & 0 & 0 & 0 & 0 & 0 \\
Sit-Stand & 2 & 95 & 3 & 0 & 0 & 0 \\
AS & 0 & 22 & 69 & 3 & 0 & 6 \\
Walk & 0 & 0 & 0 & 99 & 0 & 1 \\
Run & 0 & 0 & 0 & 0 & 100 & 0 \\
Cycle & 0 & 1 & 5 & 7 & 0 & 87 \\
Sensitivity, \% & 100 & 95 & 69 & 99 & 100 & 87 \\
Specificity, \% & 99 & 98 & 98 & 98 & 100 & 99 \\
$F$-score, \% & 100 & 96 & 81 & 99 & 100 & 93 \\
\end{tabular}

Numbers represent $\%$ of objects belonging to the true category that are classified as each classification category. Sensitivity was calculated to describe the ability to avoid false negative classifications for each activity type. Specificity was calculated to define the ability to generate true positive classifications for each activity type. $F$-score was calculated as the harmonic mean between sensitivity and positive predictive values to evaluate the overal performance of the model in classifying each activity type (26). AS, active standing; Sit-Stand, sitting or standing.
Table 2. Subject characteristics

\begin{tabular}{lcc}
\hline \hline \multicolumn{1}{c}{ Parameter } & Mean $\pm \mathrm{SD}$ & Range \\
\hline$n$ (men/women) & & $15(9 / 6)$ \\
Age, $\mathrm{yr}$ & $41 \pm 11$ & $26-59$ \\
$\mathrm{BM}, \mathrm{kg}$ & $76.6 \pm 11.4$ & $62.1-103.4$ \\
$\mathrm{Height,} \mathrm{m}$ & $1.77 \pm 0.08$ & $1.66-1.89$ \\
$\mathrm{BMI}, \mathrm{kg} / \mathrm{m}^{2}$ & $24.4 \pm 3.0$ & $19.6-29.5$ \\
$\mathrm{FM}, \mathrm{kg}$ & $20.2 \pm 6.1$ & $8.4-33.2$ \\
$\mathrm{FFM}, \mathrm{kg}$ & $56.4 \pm 7.6$ & $44.1-70.2$ \\
$\mathrm{SMR}, \mathrm{MJ} /$ day & $7.1 \pm 0.8$ & $5.7-8.3$ \\
$\mathrm{TEE}, \mathrm{MJ} /$ day & $12.5 \pm 1.9$ & $9.7-15.5$ \\
$\mathrm{AEE}, \mathrm{MJ} /$ day & $4.1 \pm 1.2$ & $2.1-6.4$ \\
$\mathrm{PAL}$ & $1.75 \pm 0.17$ & $1.43-2.06$ \\
$\mathrm{AC}$ & $228 \pm 60$ & $116-341$ \\
MET $_{\mathrm{D}}$ & $1.72 \pm 0.14$ & $1.48-1.98$ \\
\hline
\end{tabular}

BM, body mass; BMI, body mass index; FM, fat mass; FFM, fat free mass; SMR, sleeping metabolic rate; TEE, total daily energy expenditure; AEE, activity-related energy expenditure; $\mathrm{PAL}$, physical activity level; $\mathrm{AC}_{\mathrm{D}}$, daily activity counts; MET $_{\mathrm{D}}$, daily metabolic equivalent value.

$\mathrm{MET}_{\mathrm{D}}$. The independent variables considered in the regression analysis of AEE were the same as in the regression analysis of TEE with the exception of SMR. The correlation between two variables was evaluated by measuring Pearson's correlation coefficient $(R)$. Measured parameters are presented as means \pm SD. The statistical software SigmaStat (Systat software, San Jose, CA) was used for statistical analysis. The significance level was set to $P<0.05$.

\section{RESULTS}

Descriptive results. Physical characteristics of the subjects are presented in Table 2. Subjects wore the accelerometer on average $15.7 \pm 0.4 \mathrm{~h} /$ day, which was $93 \pm 5 \%$ of their waking hours. Sedentary activities like lying, sitting, and standing occupied on average $>75 \%$ of the day (Table 3 ). The average walking, running and cycling speeds of the population were $4.2 \pm 0.4,10.6 \pm 7.1$, and $20.3 \pm 6.0 \mathrm{~km} / \mathrm{h}$, respectively. The MET values selected for each activity type are presented in Table 3. According to a published compendium of physical activities (1), the intensity of lying was considered equal to the MET value of lying quietly. The intensity of sitting or standing was considered equal to the average MET value of sitting quietly, standing quietly, and sitting doing deskwork. The intensity of AS was considered equal to the MET value of multiple household tasks. The intensity of walking and running was considered equal to the MET value of walking at 2.5 $\mathrm{mile} / \mathrm{h}(\mathrm{mph})(4.0 \mathrm{~km} / \mathrm{h})$ and of running at $6.7 \mathrm{mph}(10.8$ $\mathrm{km} / \mathrm{h}$ ), respectively. The intensity of cycling was considered equal to the weighted on speed average of MET for cycling between 10 and $11.9 \mathrm{mph}(16.1$ and $19.1 \mathrm{~km} / \mathrm{h})$ and for cycling

Table 3. Types of activity performed during the day

\begin{tabular}{lccc}
\hline \hline & & \multicolumn{2}{c}{ Minutes per Day } \\
\cline { 3 - 4 } Activity Type & MET & Mean \pm SD & Range \\
\hline Lie & 1 & $513 \pm 67$ & $382-683$ \\
Sit-Stand & 1.3 & $560 \pm 111$ & $370-683$ \\
AS & 3.5 & $128 \pm 45$ & $55-231$ \\
Walk & 3 & $187 \pm 55$ & $85-291$ \\
Run & 11 & $3 \pm 4$ & $0-14$ \\
Cycle & 6.7 & $28 \pm 14$ & $8-54$ \\
\hline
\end{tabular}

MET, metabolic equivalent (1). 
between 12 and $13.9 \mathrm{mph}(19.3$ and $22.4 \mathrm{~km} / \mathrm{h}) . \mathrm{MET}_{\mathrm{D}}$ and $\mathrm{AC}_{\mathrm{D}}$ were linearly related $(R=0.90, P<0.001)$.

$P A L$ regression models. The model based on $\mathrm{AC}_{\mathrm{D}}$ explained $46 \%$ of the variation in PAL $(R=0.68, P<0.05)$, with a standard error of estimate (SEE) of 0.13 or $7.4 \%$ of the mean measured PAL (Fig. 2A). The limits of agreement between predicted and measured PAL were from -0.243 to +0.245 (Fig. $2 B$ ). The model based on $\mathrm{MET}_{\mathrm{D}}$ explained $51 \%$ of the variation in PAL $(R=0.71, P<0.05)$ with a SEE of 0.12 or $6.8 \%$ (Fig. 2C). The limits of agreement between predicted and measured PAL were from -0.233 to 0.235 (Fig. $2 D$ ). None of the physical characteristics of the population was correlated to the residual of these prediction models.

TEE regression models. The model based on SMR and $\mathrm{AC}_{\mathrm{D}}$ explained $85 \%(R=0.92)$ of the variation in TEE, with a SEE of $0.8 \mathrm{MJ} /$ day or $6.4 \%$. The model based on SMR and MET explained $87 \%(R=0.93)$ of the variation in TEE, with a SEE of $0.75 \mathrm{MJ} /$ day or $6 \%$. When basic body characteristics and $\mathrm{AC}_{\mathrm{D}}$ were used in the stepwise regression analysis, only BM and $\mathrm{AC}_{\mathrm{D}}$ were included in the prediction model, and the explained variation in TEE was $51 \%(\mathrm{R}=0.71)$, with a SEE of $1.47 \mathrm{MJ} /$ day or $11.7 \%$. The model based on BM and $\mathrm{MET}_{\mathrm{D}}$ explained $60 \%(R=0.77)$ of the variation in TEE, with a SEE of $1.33 \mathrm{MJ} /$ day or $10.6 \%$. Considering advanced body characteristics and $\mathrm{AC}_{\mathrm{D}}$, the stepwise regression analysis selected FFM and $A C_{D}$ in the prediction model of TEE. The explained variation in TEE of this model was $67 \%(R=0.82)$, with a SEE of $1.2 \mathrm{MJ} /$ day or $9.6 \%$. When advanced body characteristics and $\mathrm{MET}_{\mathrm{D}}$ were used in the stepwise regression analysis, FFM and $\mathrm{MET}_{\mathrm{D}}$ were included in the prediction model. The explained variation in TEE of this model was $76 \%(R=0.87)$, with a SEE of $1.02 \mathrm{MJ} /$ day or $8.2 \%$. None of the physical characteristics of the population was correlated to the residual of the prediction models. Coefficients, significance level, and partial correlations of all models are summarized in Table 4.
AEE regression models. When subject characteristics and $\mathrm{AC}_{\mathrm{D}}$ were entered as independent variables in a stepwise regression analysis, $\mathrm{BM}$ and $\mathrm{AC}_{\mathrm{D}}$ significantly contributed to the explained variation in AEE. The model explained $47 \%$ $(R=0.68)$ of the variation in AEE, with a SEE of $0.98 \mathrm{MJ} /$ day or $21.7 \%$. Moreover, $\mathrm{BM}$ and $\mathrm{MET}_{\mathrm{D}}$ were selected as significant predictors of AEE. The explained variation in AEE of this model was $60 \%(R=0.77)$, with a SEE of $0.85 \mathrm{MJ} /$ day or $20.7 \%$. When advanced body characteristics and $\mathrm{AC}_{\mathrm{D}}$ were used in the stepwise regression analysis, FFM and $\mathrm{AC}_{\mathrm{D}}$ were included in the prediction model. The explained variation in AEE was $60 \%(R=0.77)$, with a SEE of $0.85 \mathrm{MJ} /$ day or $20.7 \%$. Furthermore, FFM and MET $_{\mathrm{D}}$ were selected as significant predictors of AEE. This model explained $73 \%(R=0.85)$ of the variation in AEE, with a SEE of $0.70 \mathrm{MJ} / \mathrm{day}$ or $17 \%$. None of the physical characteristics of the population was correlated to the residual of the prediction models. Coefficients, significance level, and partial correlations of all models are summarized in Table 5.

\section{DISCUSSION}

This study showed that the identification of types of PA, such as lying, sitting or standing, active standing, walking, running, and cycling, performed during the day combined with a simple methodology to define activity type intensity improved the estimation of TEE, AEE, and PAL compared with activity counts. The $\mathrm{MET}_{\mathrm{D}}$ value was calculated to assess the metabolic cost of PA with the duration and the standard MET compendium value, as presented in the literature, of six common types of activity, identified with a newly developed classification tree model. $\mathrm{MET}_{\mathrm{D}}$ improved the explained variation in PAL by $5 \%$ compared with $\mathrm{AC}_{\mathrm{D}}$. Furthermore, depending on which independent variables were considered to represent differences in body size, the models based on $\mathrm{MET}_{\mathrm{D}}$
Fig. 2. Accuracy of the prediction models of the physical activity level (PAL). $A$ and $C$ : regression plots of the PAL prediction models based on activity counts per day $\left(\mathrm{AC}_{\mathrm{D}} ; A\right)$ and based on metabolic equivalent per day $\left(\mathrm{MET}_{\mathrm{D}} ; C\right) . R$, Pearson correlation coefficient of the models. $B$ and $D$ : Bland-Altman plot of the models used to predict PAL based on $\mathrm{AC}_{\mathrm{D}}$ $(B)$ and based on $\operatorname{MET}_{\mathrm{D}}(D) . P$, significance level of the association between the residual PAL and the mean PAL; CI, confidence interval.
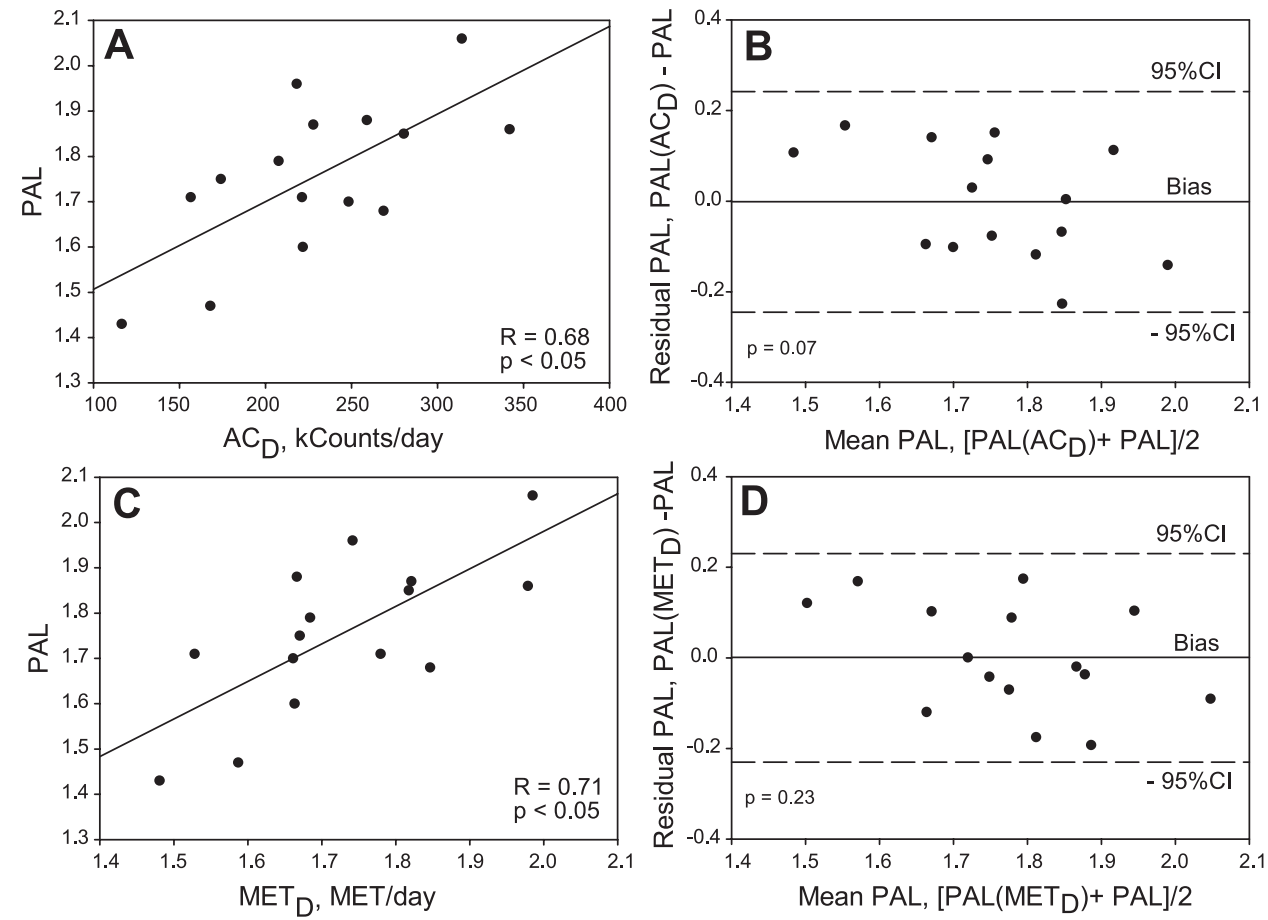

$J$ Appl Physiol • VOL $107 \cdot$ SEPTEMBER $2009 \cdot$ www.jap.org 
Table 4. Prediction models of TEE

\begin{tabular}{|c|c|c|c|c|c|c|c|c|c|}
\hline Dependent & Independent & Coefficient & $P$ & $\mathrm{p} R^{2}$ & Dependent & Independent & Coefficient & $P$ & $\mathrm{p} R^{2}$ \\
\hline \multirow[t]{3}{*}{ TEE } & INT & -9.3 & & & TEE & INT & 19.1 & & \\
\hline & SMR & 2.5 & $<0.001$ & 0.59 & & SMR & 2.5 & $<0.001$ & 0.59 \\
\hline & $\mathrm{AC}_{\mathrm{D}}$ & $1.8 \times 10^{-5}$ & $<0.001$ & 0.26 & & $\mathrm{MET}_{\mathrm{D}}$ & 8.4 & $<0.001$ & 0.28 \\
\hline Model & & & & 0.85 & Model & & & & 0.87 \\
\hline \multirow[t]{3}{*}{ TEE } & INT & 0.8 & & & TEE & INT & -8.9 & & \\
\hline & $\mathrm{BM}$ & 0.1 & $<0.05$ & 0.42 & & BM & 0.1 & $<0.05$ & 0.42 \\
\hline & $\mathrm{AC}_{\mathrm{D}}$ & $1 \times 10^{-5}$ & $<0.05$ & 0.09 & & $\mathrm{MET}_{\mathrm{D}}$ & 6.5 & $<0.05$ & 0.18 \\
\hline Model & & & & 0.51 & Model & & & & 0.60 \\
\hline \multirow[t]{3}{*}{ TEE } & INT & -2.4 & & & TEE & INT & -13.1 & & \\
\hline & FFM & 0.2 & $<0.001$ & 0.54 & & FFM & 0.2 & $<0.001$ & 0.54 \\
\hline & $\mathrm{AC}_{\mathrm{D}}$ & $1.2 \times 10^{-5}$ & $<0.05$ & 0.13 & & $\mathrm{MET}_{\mathrm{D}}$ & 7.3 & $<0.05$ & 0.22 \\
\hline Model & & & & 0.67 & Model & & & & 0.76 \\
\hline
\end{tabular}

$P$, significance level; $\mathrm{p} R^{2}$, partial correlation; Model, $R^{2}$ of the prediction model; INT, intercept.

improved the explained variation in TEE from $2 \%$ to $9 \%$ and improved the explained variation in $\mathrm{AEE}$ by $13 \%$, compared with the models based on $\mathrm{AC}_{\mathrm{D}}$.

Only a small number of accelerometers have been validated against the gold standard technique of double-labeled water. Those that were validated often showed poor correlations with energy expenditure or the main contribution to the explained variation in TEE, or AEE was determined by subjects' physical characteristics (21). Very few studies reported a higher accuracy in predicting TEE, AEE, and PAL than that of the models obtained in the present study $(5,20,21)$. Plasqui et al. (20) developed a prediction model of TEE using SMR and $\mathrm{AC}_{\mathrm{D}}$ as independent variables. The explained variation of the model was $90 \%$. In our model based on the same independent variables, the explained variation in TEE was $85 \%$. Carter et al. (5) developed a model to predict TEE using body height and $\mathrm{AC}_{\mathrm{D}}$ in a population of young male adults as independent variables. The explained variation of the model was $73 \%$, and $\mathrm{AC}_{\mathrm{D}}$ accounted for $27 \%$ of the explained variation in TEE. Plasqui et al. (20) developed a model to predict TEE in a population of young adults using age, $\mathrm{BM}$, height, and $\mathrm{AC}_{\mathrm{D}}$ as independent variables. The explained variation of the model was $83 \%$, and $\mathrm{AC}_{\mathrm{D}}$ accounted for $19 \%$ to the explained variation in TEE. In our study, TEE was predicted by $\mathrm{BM}$ and $\mathrm{AC}_{\mathrm{D}}$. This model explained $51 \%$ of the variation in TEE, while $\mathrm{AC}_{\mathrm{D}}$ accounted for $9 \%$ of the explained variation in TEE. Although comparing these prediction models is difficult because of the different independent variables included in the regression, it appeared that those developed in the present study showed a lower explained variance in TEE. Additionally, the contribution of $\mathrm{AC}_{\mathrm{D}}$ to explain the variation in TEE was lower. This was also observed in the models to predict AEE and PAL compared with the study of Plasqui et al. (20). A limitation of this study was the fact that the habitual PA was determined during a monitoring period of 5 days, while the TEE was assessed over a period of $2 \mathrm{wk}$, according to the double-labeled water protocol. This could have determined a decrease in the contribution of $\mathrm{AC}_{\mathrm{D}}$ to the explained variation in TEE, AEE, and PAL, because of a reduced ability of $\mathrm{AC}_{\mathrm{D}}$ to describe PA. However, some studies have shown that as little as 3-4 days of monitoring was sufficient to achieve a reliability of $>80 \%$ in measurements of PA with accelerometers $(15,17)$. In the study of Plasqui et al. (20) the activity monitor was equipped with a piezo-electric acceleration sensor, while in the present study the Tracmor was equipped with a piezo-capacitive sensor that allowed the identification of postures by detecting static accelerations. Additional research is required to understand whether the use of piezo-capacitive acceleration sensors determined a decrease in the ability of $\mathrm{AC}_{\mathrm{D}}$ to account for the explained variance in TEE, $\mathrm{AEE}$, and PAL compared with the $\mathrm{AC}_{\mathrm{D}}$ measured with activity monitors equipped with piezo-electric sensors. Furthermore, different data processing of the acceleration signal should be also carefully considered as a confounding factor when comparing the ability of piezo-electric and piezo-capacitive sensors in measuring PA.

The $\mathrm{MET}_{\mathrm{D}}$ value provided a more accurate assessment of PA compared with $\mathrm{AC}_{\mathrm{D}}$, since the developed model to predict TEE, AEE, and PAL showed a higher accuracy. The calculation of $\mathrm{MET}_{\mathrm{D}}$ was based on the use of a newly developed classification algorithm for the identification of types of physical activity performed during the day. The assessment of PA by identifying activity types was hypothesized to improve the estimation of energy expenditure. This assumption was based on the evidence that the relation

Table 5. Prediction models of AEE

\begin{tabular}{|c|c|c|c|c|c|c|c|c|c|}
\hline Dependent & Independent & Coefficient & $P$ & $\mathrm{p} R^{2}$ & Dependent & Independent & Coefficient & $P$ & $\mathrm{p} R^{2}$ \\
\hline & BM & 0.05 & $<0.05$ & 0.26 & & BM & 0.07 & $<0.05$ & 0.35 \\
\hline Model & & & & 0.47 & Model & & & & 0.60 \\
\hline \multirow[t]{3}{*}{ AEE } & INT & -4.9 & & & AEE & INT & -14.7 & & \\
\hline & FFM & 0.1 & $<0.05$ & 0.21 & & FFM & 0.12 & $<0.001$ & 0.48 \\
\hline & $\mathrm{AC}_{\mathrm{D}}$ & $1.3 \times 10^{-5}$ & $<0.05$ & 0.38 & & $\mathrm{MET}_{\mathrm{D}}$ & 7.1 & $<0.001$ & 0.25 \\
\hline
\end{tabular}

$P$, significance level; $\mathrm{p} R^{2}$, partial correlation; Model, $R^{2}$ of the prediction model; INT, intercept. 
between energy expenditure and accelerometer output depends on the type of activity performed. A few studies (19, 27) have shown that different linear equations could be developed to estimate the MET of activities such as sitting, standing, walking, and housework, using activity counts. Furthermore, a unique linear relationship between activity counts and activity intensity is not suitable for both running and cycling activities. In fact, these two activities generate a diverse amount of activity counts even at a similar level of METs. In this study, the MET $_{\mathrm{D}}$ value accounted for the different contribution of six activity types to TEE, AEE, and PAL. This was possible because the assessment of activity intensity was independent from activity counts. The intensity of lying, sitting or standing, and AS was assumed to be equal to a specific MET value as obtained from a published compendium of PA (1). The intensity of each walking, running, and cycling activity was assumed to be equal to the MET value of walking at $2.5 \mathrm{mph}$, running at $6.7 \mathrm{mph}$, and cycling between 10 and $13.9 \mathrm{mph}$, because these MET values were representative of the activity intensity at the average speed measured during the monitoring period. The only independent variable determining $\mathrm{MET}_{\mathrm{D}}$ was the daily duration of the six activity types, since activity intensity was considered constant. This might allow the applicability of the prediction models based on $\mathrm{MET}_{\mathrm{D}}$ to any method able to accurately detect the daily duration of the types of activity identified in this study. However, a methodology that allows the detection of activity intensity for each activity type and for each activity bout could be considered to further improve the estimation accuracy of TEE, AEE, and PAL. The challenge would be represented mainly by the determination of intensity for sedentary and unspecified dynamic activities, such as Sit-Stand or AS, which occupy a large part of the daytime and could importantly contribute to the definition of the metabolic cost of PA (27).

In the literature, some attempts have been made to improve accelerometer-based estimation of energy expenditure by defining a specific regression equation to relate the metabolic cost of PA to activity counts for specific groups of activities such as locomotive and lifestyle activities (7) or sedentary, locomotive, or housework activities $(19,27)$. Additionally, nonlinear models such as artificial neural networks have been applied to the raw acceleration of the body to improve the prediction accuracy of energy expenditure $(6,23)$. However, none of these computationally sophisticated techniques has been validated yet in free-living conditions by using, as a reference measure of energy expenditure, double-labeled water. In this study, PA was assessed by a $\mathrm{MET}_{\mathrm{D}}$ parameter that accounted for the different contribution to the metabolic cost of PA of each identified type of activity. This approach was similar to that implemented in the ActiReg activity monitor to estimate TEE (11). The ActiReg includes two accelerometers. They are positioned on the chest and on the thigh to determine body posture and to categorize PA in three classes of intensity. Depending on the posture and on the activity intensity, a MET value is used to describe the energy cost of PA. Thus the definition of energy expenditure was derived from information on posture (lying, sitting, and standing) and the intensity of PA. The ActiReg has been validated against double-labeled water, and a standard error of $1.24 \mathrm{MJ} / \mathrm{day}$ was obtained in the estimation of TEE (11). Therefore, the prediction accuracy was poorer than that achieved by the models developed using $\operatorname{MET}_{\mathrm{D}}$.

In this study, PAL and AEE were calculated from measurements of TEE and SMR. In the literature, TEE is often corrected by resting metabolic rate (RMR) to determine PAL and AEE. The choice of using SMR instead of RMR derived from the fact that measurements of SMR showed a high reproducibility. Indeed, the intraindividual coefficient of variation of SMR measured in a respiration chamber has been estimated to be below 2\% (24). Considering that SMR is $\sim 5 \%$ lower than RMR (13), the mean values of PAL and AEE measured in this study were systematically higher than those derived from TEE and RMR. However, the variability in PAL and AEE was not significantly affected by the use of SMR instead of RMR. Thus the estimation accuracy of the models to predict PAL and AEE was not influenced by the selection of SMR as correction factor for TEE.

In conclusion, identification of activity types combined with standard MET compendium values improved the assessment of energy expenditure compared with activity counts. Future studies could focus on the development of models to objectively measure the intensity of common types of PA to further increase the accuracy of the energy expenditure estimation.

\section{DISCLOSURES}

This work was funded by Philips Research.

\section{REFERENCES}

1. Ainsworth BE, Haskell WL, Whitt MC, Irwin ML, Swartz AM, Strath SJ, O'Brien WL, Bassett DR Jr, Schmitz KH, Emplaincourt PO, Jacobs DR Jr, Leon AS. Compendium of physical activities: an update of activity codes and MET intensities. Med Sci Sports Exerc 32: S498-S516, 2000.

2. Bland JM, Altman DG. Statistical methods for assessing agreement between two methods of clinical measurement. Lancet 1: 307-310, 1986.

3. Bonomi AG, Goris AHC, Yin B, Westerterp KR. Detection of type, duration and intensity of physical activity using an accelerometer. Med Sci Sports Exerc. In press. (doi:10.1249/MSS.0b013e3181a24536).

4. Bouten CVC, Koekkoek KTM, Verduin M, Kodde R, Janssen JD. A triaxial accelerometer and portable data processing unit for the assessment of daily physical activity. IEEE Trans Biomed Eng 44: 136-147, 1997.

5. Carter J, Wilkinson D, Blacker S, Rayson M, Bilzon J, Izard R, Coward A, Wright A, Nevill A, Rennie K, McCaffrey T, Livingstone B. An investigation of a novel three-dimensional activity monitor to predict free-living energy expenditure. J Sports Sci 26: 553-561, 2008.

6. Chen KY, Sun M. Improving energy expenditure estimation by using a triaxial accelerometer. J Appl Physiol 83: 2112-2122, 1997.

7. Crouter SE, Clowers KG, Bassett DR. A novel method for using accelerometer data to predict energy expenditure. J Appl Physiol 100: 1324-1331, 2006.

8. Ekelund U, Sjostrom M, Yngve A, Poortvliet E, Nilsson A, Froberg K, Wedderkopp N, Westerterp K. Physical activity assessed by activity monitor and doubly labeled water in children. Med Sci Sports Exerc 33: 275-281, 2001.

9. Ermes M, Parkka J, Mantyjarvi J, Korhonen I. Detection of daily activities and sports with wearable sensors in controlled and uncontrolled conditions. IEEE Trans Inf Technol Biomed 12: 20-26, 2008.

10. Frank E, Hall M, Holmes G, Kirkby R, Pfahringer B, Witten IH, Trigg $\mathbf{L}$. Weka - a machine learning workbench for data mining. In: Data Mining and Knowledge Discovery Handbook. Heidelberg: Springer, 2005, p. 1305-1314.

11. Hustvedt BE, Christophersen A, Johnsen LR, Tomten H, McNeill G, Haggarty P, Lovo A. Description and validation of the ActiReg: a novel instrument to measure physical activity and energy expenditure. Br J Nutr 92: 1001-1008, 2004.

12. Karantonis DM, Narayanan MR, Mathie M, Lovell NH, Celler BG. Implementation of a real-time human movement classifier using a triaxial accelerometer for ambulatory monitoring. IEEE Trans Inf Technol Biomed 10: 156-167, 2006. 
13. Kumahara H, Yoshioka M, Yoshitake Y, Shindo M, Schutz Y, Tanaka H. The difference between the basal metabolic rate and the sleeping metabolic rate in Japanese. J Nutr Sci Vitaminol (Tokyo) 50: 441-445, 2004.

14. Leenders N, Sherman WM, Nagaraja HN, Kien CL. Evaluation of methods to assess physical activity in free-living conditions. Med Sci Sports Exerc 33: 1233-1240, 2001.

15. Levin S, Jacobs DR, Ainsworth BE, Richardson MT, Leon AS. Intra-individual variation and estimates of usual physical activity. Ann Epidemiol 9: 481-488, 1999.

16. Macfarlane DJ, Lee CCY, Ho EYK, Chan KL, Chan D. Convergent validity of six methods to assess physical activity in daily life. $J$ Appl Physiol 101: 1328-1334, 2006.

17. Matthews CE, Ainsworth BE, Thompson RW, Bassett DR. Sources of variance in daily physical activity levels as measured by an accelerometer. Med Sci Sports Exerc 34: 1376-1381, 2002.

18. Melanson EL, Freedson PS. Physical activity assessment: a review of methods. Crit Rev Food Sci Nutr 36: 385-396, 1996.

19. Midorikawa T, Tanaka S, Kaneko K, Koizumi K, Ishikawa-Takata K, Futami J, Tabata I. Evaluation of low-intensity physical activity by triaxial accelerometry. Obesity (Silver Spring) 15: 3031-3038, 2007.

20. Plasqui G, Joosen A, Kester AD, Goris AHC, Westerterp K. Measuring free-living energy expenditure and physical activity with triaxial accelerometry. Obes Res 13: 1363-1369, 2005.

21. Plasqui G, Westerterp KR. Physical activity assessment with accelerometers: an evaluation against doubly labeled water. Obesity (Silver Spring) 15: 2371-2379, 2007.

22. Pober DM, Staudenmayer J, Raphael C, Freedson PS. Development of novel techniques to classify physical activity mode using accelerometers. Med Sci Sports Exerc 38: 1626-1634, 2006.
23. Rothney MP, Neumann M, Beziat A, Chen KY. An artificial neural network model of energy expenditure using nonintegrated acceleration signals. J Appl Physiol 103: 1419-1427, 2007.

24. Schoffelen PFM, Westerterp KR, Saris WHM, TenHoor F. A dualrespiration chamber system with automated calibration. J Appl Physiol 83: 2064-2072, 1997.

25. Siri WE. Body composition from fluid space and density: analysis of methods. Nutrition 9: 481-491, 1993.

26. Sokolova M, Japkowicz N, Szpakowicz S. Beyond accuracy, f-score and ROC: a family of discriminant measures for performance evaluation. In: AI 2006: Advances in Artificial Intelligence 19th Australian Joint Conference on Artificial Intelligence Proceedings, edited by Sattar A, Kang BH. Berlin: Springer, 2006, p. 1015-1021.

27. van Hees VT, van Lummel RC, Westerterp KR. Estimating activityrelated energy expenditure under sedentary conditions using a tri-axial seismic accelerometer. Obesity (Silver Spring) 17: 1287-1292, 2009.

28. Veltink PH, Bussmann HB, de Vries W, Martens WL, Van Lummel RC. Detection of static and dynamic activities using uniaxial accelerometers. IEEE Trans Rehabil Eng 4: 375-385, 1996.

29. Weir JBD. New methods for calculating metabolic rate with special reference to protein metabolism. J Physiol 109: 1-9, 1949.

30. Westerterp KR. Physical activity as determinant of daily energy expenditure. Physiol Behav 93: 1039-1043, 2008.

31. Westerterp KR, Wouters L, Lichtenbelt WDV. The Maastricht protocol for the measurements of body composition and energy expenditure with labeled water. Obes Res 3: 49-57, 1995.

32. Zhang K, Werner P, Sun M, Pi-Sunyer FX, Boozer CN. Measurement of human daily physical activity. Obes Res 11: 33-40, 2003. 